\title{
Regularization Method for Nonlinear Singularly Perturbed Systems of Fractional Order
}

\author{
Burkhan Kalimbetov ${ }^{1, *}$, Riskeldy Turgunbaev² \\ ${ }^{1}$ Department of Mathematics, Akhmed Yasawi University \\ Turkestan, Kazakhstan, \\ ${ }^{2}$ Department of Mathematics, Tashkent State Pedagogical University \\ Tashkent, Uzbekiztan, \\ *Corresponding author's email: burkhan.kalimbetov [AT] ayu.edu.kz
}

\begin{abstract}
The paper is devoted to construction of an asymptotic solution of a weakly nonlinear singularly perturbed differential system of fractional order. To construct the asymptotic solution we use ideas of normal differential forms method of V.F. Safonov. In the environment of the computer mathematical system Maple, approximate solutions are calculated, and corresponding solution schedules for various values of a small parameter are constructed.
\end{abstract}

Keywords--- Nonlinear differential equation of fractional order, regularization, asymptotic, iteration problems, normal and unique solvability, computer mathematics system Maple.

\section{INTRODUCTION}

We consider a nonlinear problem of the form

$$
\varepsilon y^{(1 / 2)}(t, \varepsilon)=A(t) y+\varepsilon f(t, y)+h(t), \quad y(0, \varepsilon)=y^{0}, t \in[0, T],
$$

where $y=\left\{y_{1}, \ldots, y_{n}\right\}$ unknown vector function, $A(t)$ known $n \times n$ matrix-function, $f(t, y)=\left\{f_{1}, \ldots, f_{n}\right\}$ known vector-function, $y^{0}=\left\{y_{1}^{0}, \ldots, y_{n}^{0}\right\} \in \square^{n}$ known constant vector, $\varepsilon>0$ small parameter. We look for an asymptotic solution of the problem (1) on the following bounds:

1) $A(t) \in C^{\infty}\left([0, T], \square^{n}\right), h(t) \in C^{\infty}\left([0, T], \square^{n}\right)$;

2) spectrum $\left\{\lambda_{j}(t)\right\} \equiv \sigma(A(t))$ of the matrix-function $A(t)$ satisfies the requirements:

i) $\lambda_{j}(t) \neq 0 \quad \forall t \in[0, T], \quad j=\overline{1, n}$;

ii) $\lambda_{i}(t) \neq \lambda_{j}(t) \quad \forall t \in[0, T], i \neq j, i, j=\overline{1, n}$;

iii) $\operatorname{Re} \lambda_{j}(t) \leq 0 \quad \forall t \in[0, T], j=\overline{1, n}$.

The problem (1) will be considered in general for non-linearity $f(t, y)$, analytical with respect to $y$, i.e. the function $f(t, y)$ belongs to a class $\Omega$, which is described as follows:

Definition 1. They say that the vector functions $f(t, y)=\left\{f_{1}, f_{2}, \ldots, f_{n}\right\}$ belong to the class $\Omega$, if its each component $f_{i}(t, y)$ is expanded into the series:

$$
f_{i}(t, y)=\sum_{|m| \geq 0} f_{i}^{(m)}(t) y^{m} \equiv \sum_{m_{1}+\ldots+m_{n}=0}^{\infty} f_{i}^{\left(m_{1}, \ldots, m_{n}\right)}(t) y_{1}^{m_{1}} \ldots y_{n}^{m_{n}},
$$

where all coefficients $f_{i}^{(m)}(t) \in C^{\infty}\left([0, T], \square^{1}\right)$ converge absolutely and uniformly with respect to $t \in[0, T]$ in the polycylinder $\quad \Pi=\left\{y:\left|y_{i}\right|<R, j=\overline{1, n}\right\} \quad$ (here: $\quad m=\left(m_{1}, \ldots, m_{n}\right) \quad$ multiindex, $|m|=m_{1}+\ldots+m_{n}, y^{m}=y_{1}^{m_{1}} \ldots y_{n}^{m_{n}}, R>0$ some constant.

Wenotethat absolute and uniform convergence of the series (2) in the domain $\Pi$ (or another similar series) means that the series $\sum_{|m| \geq 0}\left|f_{i}^{(m)}(t)\right|\left|y^{m}\right|$ converges uniformly with respect to $t \in[0, T]$ at each fixed $y \in \Pi$. 


\section{REGULARIZATION OF PROBLEM}

Regularization of the problem (1) is performed using the vector $u=\left\{u_{1}, \ldots, u_{n}\right\}$ of regularizing variables

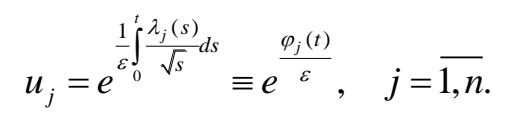

These variables satisfy the linear differential system $\varepsilon \dot{u}=\Lambda(t) u, u(0, \varepsilon)=\overline{1} \equiv\{1, \ldots, 1\}$ (linear normal form). For the function $\tilde{y}(t, u, \varepsilon)$ such that

$$
\left.\tilde{y}(t, u, \varepsilon)\right|_{u=u(t, \varepsilon)} \equiv y(t, \varepsilon)
$$

where $u=u(t, \varepsilon) \equiv\left\{e^{\frac{\varphi_{1}(t)}{\varepsilon}}, \ldots, e^{\frac{\varphi_{n}(t)}{\varepsilon}}\right\}, y(t, \varepsilon)$ exact solution of the system (1), it is naturally to put the following problem:

$$
\varepsilon \sqrt{t} \frac{\partial \tilde{y}}{\partial t}+\frac{\partial \tilde{y}}{\partial u} \Lambda(t) u-A(t) \tilde{y}-\varepsilon f(t, \tilde{y})=h(t), \quad \tilde{y}(0, \overline{1})=y^{0} .
$$

Solution of the "extended" problem (3) is defined as a series

$$
\tilde{y}(t, u, \varepsilon)=\sum_{k=0}^{\infty} \varepsilon^{k} y_{k}(t, u)
$$

by non negative powers of the parameter $\varepsilon$. Putting this series into the problem (3), and equating coefficients at the same degrees $\varepsilon$, we obtain the following iteration problems:

$$
\begin{array}{rlrl}
L y_{0}(t, \tau) & \equiv \frac{\partial y_{0}}{\partial u} \Lambda(t) u-A(t) y_{0}=h(t), & y_{0}(0, \overline{1})=y^{0} ; \\
L y_{1}(t, \tau) & =-\sqrt{t} \frac{\partial y_{0}}{\partial t}+f\left(t, y_{0}\right) \quad y_{1}(0, \overline{1})=0 ; \\
L y_{2}(t, u) & =-\sqrt{t} \frac{\partial y_{1}}{\partial t}+\frac{\partial f\left(t, y_{0}\right)}{\partial y} y_{1}, & y_{2}(0, \overline{1})=0 ; \\
L y_{k}(t, u)=-\sqrt{t} \frac{\partial y_{k-1}}{\partial t}+P_{k}\left(t, y_{0}, y_{1}, \ldots, y_{k-1}\right), & y_{k-1}(0, \overline{1})=0, k \geq 3,
\end{array}
$$

where $P_{k}\left(t, y_{0}, \ldots, y_{k-1}\right)$ some polynomial of $y_{1}, \ldots, y_{k-1}$ with coefficients, that depend on partial derivatives of functions $f(t, y)$ at the point $y=y_{0}(t, u)$, moreover, $P_{k}\left(t, y_{0}, \ldots, y_{k-1}\right)$ is linear with respect to the last argument $y_{k-1}$.

Iteration problems $\left(5_{\mathrm{\kappa}}\right)$ will be solved in the space $U$ of vector functions $w(t, u)=\left\{w_{1}(t, u), w_{2}(t, u), \ldots, w_{n}(t, u)\right\}$, each component of which is represent table by the series (2) with coefficients $w_{i}(t, u) \in C^{\infty}\left([0, T], \square^{1}\right)$, converging absolutely and uniformly with respect to $t \in[0, T]$ in the domain $G=\left\{u:\left|u_{i}\right|<1+\delta, j=\overline{1, n}\right\}$, where $\delta>0$ some (small) constant.

\section{SOLVABLE OF FIRST ITERATION PROBLEM}

The iteration problem $\left(5_{0}\right)$ in the space $U$ has the following solution:

$$
\begin{aligned}
y_{0}(t, u) & =\xi_{1}^{(0)}(t) c_{1}(t) u_{1}+\xi_{2}^{(0)}(t) c_{2}(t) u_{2}+\ldots+\xi_{n}^{(0)}(t) c_{n}(t) u_{n}- \\
& -A^{-1}(t) h(t) \equiv C(t) \operatorname{diag}\left(\xi_{1}^{(0)}(t), \xi_{2}^{(0)}(t), \ldots, \xi_{n}^{(0)}(t)\right) u-A^{-1}(t) h(t),
\end{aligned}
$$

where $\xi_{j}^{(0)}(t) \in C^{\infty}\left([0, T], \square^{1}\right)$ arbitrary functions, $c_{j}(t)$ eigenvectors of the matrix $A(t)$ corresponding to eigenvalues $\lambda_{j}(t), j=\overline{1, n}, C(t) \equiv\left(c_{1}(t), c_{2}(t), \ldots, c_{n}(t)\right)$. Subjecting this solution to the initial condition $y_{0}(0, \overline{1})=y^{0}$, we will have

where we find the values $\xi_{j}^{(0)}(0)$ :

$$
\xi_{1}^{(0)}(0) c_{1}(0)+\xi_{2}^{(0)}(0) c_{2}(0)+\ldots+\xi_{n}^{(0)}(0) c_{n}(0)=A^{-1}(t) h(t)+y^{0},
$$

$$
\xi_{j}^{(0)}(0)=\left(A^{-1}(0) h(0)+y^{0}, d_{j}(0)\right), \quad j=\overline{1, n},
$$


where $d_{j}(t)$ eigenvectors of the matrix $A^{*}(t)$, corresponding to eigenvalues $\bar{\lambda}_{j}(t), j=\overline{1, n}$, (i.e. $\left.A^{*}(t) d_{j}(t) \equiv \bar{\lambda}_{j}(t) d_{j}(t), j=\overline{1, n}\right)$. If now we put the function (6) to $f\left(t, y_{0}\right)$, then we obtain the series:

$$
f\left(t, y_{0}(t, u)\right)=\sum_{|m| \geq 0} f^{(m)}\left(t, \xi^{(0)}(t)\right) u^{m},
$$

belonging (at some auxiliary conditions) to the class $U$. We now introduce the following designation: if $w(t, u)$ series $\sum_{|m| \geq 0} w^{(m)}(t) u^{m}$, then by $w^{(k)}(t, u)$ we will denote the sum of the members of this dimension series $|m|=k$, i.e.

$$
w^{(k)}(t, u)=\sum_{|m|=k} w^{(m)}(t) u^{m}, k=0,1,2, \ldots,
$$

and by $U^{(k)} \subset U$ subspace of the class $U$, consisting of all sorts of such sums (assume that the element $0 \equiv \sum_{|m|=k} 0 \cdot u^{m}$ belongs to the space $\left.U^{(k)}\right)$. In the space $U^{(k)}$ we define the dot (at any $t \in[0, T]$ ) product:

$$
\begin{gathered}
<w^{(k)}(t, u), z^{(k)}(t, u)>\equiv<\sum_{|m|=k} w^{(k)}(t) u^{m}, z^{(k)}(t) u^{m}>\stackrel{\text { def }}{=} \\
\stackrel{\text { def }}{=} \sum_{|m|=k}\left(w^{(k)}(t), z^{(k)}(t)\right)=\sum_{|m|=k}\left(\left(w^{(k)}(t)\right)^{T} \overline{z^{(k)}(t)} .\right.
\end{gathered}
$$

Highlight in (8) $f^{(1)}\left(t, y_{0}(t, u)\right)$. Denoting $\bar{y}(t)=-A^{-1}(t) h(t)$ and expanding $f(t, y)$ into Taylor series in a neighborhood of the point $y=\bar{y}(t)$, we will have:

$$
f(t, y)=f(t, \bar{y}(t))+\frac{\partial f(t, \bar{y}(t))}{\partial u}(y-\bar{y}(t))+\ldots
$$

where dots denote the members of the dimension $|m| \geq 2$ relatively to $y-\bar{y}(t)$. Putting here $y=y_{0}(t, u)$ from (6), we get

where we find

$$
f\left(t, y_{0}(t, u)\right)=f(t, \bar{y}(t))+\frac{\partial f(t, \bar{y}(t))}{\partial u} C(t) \operatorname{diag}\left(\xi_{1}^{(0)}(t), \ldots, \xi_{n}^{(0)}(t)\right) u+\ldots
$$

$$
\begin{aligned}
f^{(1)}\left(t, y_{0}(t, u)\right) & =\frac{\partial f(t, \bar{y}(t))}{\partial y} C(t) \operatorname{diag}\left(\xi_{1}^{(0)}(t), \ldots, \xi_{n}^{(0)}(t)\right) u \equiv \\
& \equiv \sum_{j=1}^{n} \frac{\partial f(t, \bar{y}(t))}{\partial y} c_{j}(t) \xi_{j}^{(0)}(t) u_{j} .
\end{aligned}
$$

It will be shown below that for solvability of the system $\left(5_{1}\right)$ in the space $U$, it is necessary and sufficient the conditions:

$$
<-\sqrt{t} \frac{\partial y_{0}^{(1)}(t, u)}{\partial t}+f^{(1)}(t, u), d_{j}(t) u_{j}>\equiv 0 \quad \forall t \in[0, T], j=\overline{1, n} .
$$

Putting here functions (6) and (9), we will have

$$
<-\sum_{j=1}^{n}\left[\sqrt{t}\left(\xi_{j}^{(0)}(t) c_{j}(t)\right)^{\bullet} u_{j}+\frac{\partial f(t, \bar{y}(t))}{\partial y} c_{j}(t) \xi_{j}^{(0)}(t) u_{j}\right], d_{j}(t) u_{j}>\equiv 0 \forall t \in[0, T],
$$

or

$$
-\sqrt{t} \dot{\xi}_{j}^{(0)}(t)+\left(\frac{\partial f(t, \bar{y}(t))}{\partial y} c_{j}(t)-\dot{c}_{j}(t), d_{j}(t)\right) \xi_{j}^{(0)}(t) \equiv 0 \quad j=\overline{1, n} .
$$

Joining the initial conditions (7) to these equations, we find uniquely the functions $\xi_{j}^{(0)}(t)$ :

$$
\xi_{j}^{(0)}(t)=\left(A^{-1}(0) h(0)+y^{0}, d_{j}(0)\right) \cdot e^{\int^{t} \frac{\left(\frac{\partial f(s, \bar{y}(s))}{\partial y} c_{j}(s)-\dot{c}_{j}(s), d_{j}(s)\right.}{\sqrt{s}} d s}, j=\overline{1, n},
$$

thus, we construct the solution (6) of the problem (50) in the space $U$ unambiguously. 
Let us turn to solution of the next problems $\left(5_{\mathrm{K}}\right) k \geq 1$. However, for this we need information from the theory of solvability of general form systems $L y(t, u)=h(t, u)$ in the space $U$. Turning to the presentation of this theory, we note that the operator $L^{*}$ in the space $U^{(1)}$ will have the form $L^{*}=\sum_{j=1}^{n} \bar{\lambda}_{j}(t) u_{j} \frac{\partial}{\partial u_{j}}$ and the basis of its kernel $\operatorname{Ker} L^{*}$ in $U^{(1)}$ will be: $v_{j}(t, u)=d_{j}(t) u_{j},(j=\overline{1, n})$ that it is easy to see directly.

\section{NORMAL AND UNIQUE SOLVABILITY OF GENERAL ITERATION PROBLEM}

Each of the iteration problems $\left(5_{\mathrm{K}}\right)$ can be written as follows:

$$
L y(t, u) \equiv \sum_{j=1}^{n} \lambda_{j}(t) u_{j} \frac{\partial y}{\partial u_{j}}-A(t) y=h(t, u), \quad y_{0}(0, \overline{1})=y^{*},
$$

where $h(t, u)$ corresponding right hand side, $y^{*} \in \square^{n}$ known constant vector. The following proposition holds.

Theorem 1. Let the right hand side $h(t, u) \in U$ and the conditions 1), 2a) $-2 \mathrm{~b}$ ) hold. Then for solvability of the system (11) in $U$ it is necessary and sufficient that

$$
<h^{(1)}(t), v_{j}(t, u)>\equiv 0 \quad \forall t \in[0, T, \quad j=\overline{1, n} .
$$

Proof. Let $h(t, u) \in U$ have the form $h(t, u)=\sum_{|m| \geq 0} h^{(m)}(t) u^{m}$. We will define o solution of the system (11) in the form of the series

$$
y(t, u)=\sum_{|m| \geq 0} y^{(m)}(t) u^{m} .
$$

Putting this series in to the system (11), we will have the formal equality:

$$
\sum_{|m| \geq 0} y^{(m)}(t) \sum_{j=1}^{n} \lambda_{j}(t) u_{j} \frac{\partial u^{m}}{\partial u_{j}}-\sum_{|m| \geq 0} A(t) y^{(m)}(t) u^{m}=\sum_{|m| \geq 0} h^{(m)}(t) u^{m} .
$$

Taking into account that $\frac{\partial u^{m}}{\partial u_{j}}=m_{j} u^{m-e_{j}} \equiv m_{1} u_{1}^{m_{1}} \ldots u_{j}^{m j-1} \ldots u_{n}^{m_{n}}$, we obtain:

$$
\sum_{|m| \geq 0}\left(\sum_{j=1}^{n} m_{j} \lambda_{j}\right) y^{(m)}(t) u^{m}-\sum_{|m| \geq 0} A(t) y^{(m)}(t) u^{m}=\sum_{|m| \geq 0} h^{(m)}(t) u^{m} .
$$

Equating here coefficients at the same powers $u^{m}$, we get the system

$$
[(m, \lambda(t)) I-A(t)] u^{(m)}(t)=h^{(m)}(t)|m| \geq 0 .
$$

Defining solution of this system as $y^{(m)}(t)=C(t) \xi^{(m)}(t)$, we obtain the equations systems:

$$
[(m, \lambda(t)) I-\Lambda(t)] \xi^{(m)}(t)=C^{-1}(t) h^{(m)}(t)|m| \geq 0,
$$

which can be rewritten in the scalar form:

$$
\left[(m, \lambda(t))-\lambda_{j}(t)\right] \xi_{j}^{(m)}(t)=\left(h^{(m)}(t), d_{j}(t)\right), \quad j=\overline{1, n}, \quad|m| \geq 0 .
$$

At $|m|=0$ we find:

$$
\xi_{j}^{(m)}(t)=\frac{\left(h^{(0)}(t), d_{j}(t)\right)}{-\lambda_{j}(t)}, \quad j=\overline{1, n},
$$

and when $|m| \geq 2$ we calculate:

$$
\xi_{j}^{(m)}(t)=\frac{\left(h^{(m)}(t), d_{j}(t)\right)}{(m, \lambda(t))-\lambda_{j}(t)}, \quad j=\overline{1, n} .
$$

When $|m|=1$ we have the system $\left(e_{k}=\{0, \ldots, 1, \ldots, 0\}, k-\right.$ th ort in $\left.\square^{n}\right)$ 


$$
\left(\lambda_{k}(t)-\lambda_{j}(t)\right) \xi_{j}^{e_{k}}(t)=\left(h_{j}^{e_{k}}(t), d_{j}(t)\right), \quad j=\overline{1, n},
$$

$\left(k \in\{1,2, \ldots, n\}\right.$ fixed). For solvability of this system in the space $C^{\infty}\left([0, T], \square^{n}\right)$ it is necessary and sufficient that

$$
\left(h_{j}^{e_{k}}(t), d_{k}(t)\right) \equiv 0 \quad \forall t \in[0, T] .
$$

Moreover, $\xi_{k}^{e_{k}}(t) \in C^{\infty}\left([0, T], \square^{1}\right) \backslash$ arbitrary function, and $\xi_{j}^{e_{k}}(t)$ at $k \neq j$ can be calculated uniquely:

therefore

$$
\xi_{j}^{e_{k}}(t)=\frac{\left(h^{e_{k}}(t), d_{j}(t)\right)}{\lambda_{k}(t)-\lambda_{j}(t)}, j=\overline{1, n}, j \neq k,
$$

$$
y^{e_{k}}(t)=C(t) \xi^{e_{k}}=\xi_{j}^{e_{k}}(t) c_{k}(t)+\sum_{j \neq k, j=1}^{n} \frac{\left(h^{e_{k}}(t), d_{j}(t)\right)}{\lambda_{k}(t)-\lambda_{j}(t)} c_{j}(t) .
$$

Hence, for solvability of the system (11) in the class of formal power series (13) it is necessary and sufficient that conditions (18) are simultaneously satisfied for all $k=\overline{1, n}$, which are equivalent to conditions (12). In this case, the formal solution (13) has the form:

$$
\begin{gathered}
y(t, u)=\sum_{k=1}^{n} y^{e_{k}}(t) u^{e_{k}}+\sum_{|m| \geq 0,|m| \neq 1} y^{(m)}(t) u^{m}=\sum_{k=1}^{n}\left[\xi_{k}(t) c_{k}(t)+\right. \\
\left.+\sum_{j \neq k, j=1}^{n} \frac{\left(h^{e_{k}}(t), d_{j}(t)\right)}{\lambda_{k}(t)-\lambda_{j}(t)} c_{j}(t)\right] u_{k}+\sum_{|m| \geq 0,|m| \neq 1}\left(\sum_{j=1}^{n} \frac{\left(h^{(m)}(t), d_{j}(t)\right)}{(m, \lambda(t))-\lambda_{j}(t)} c_{j}(t)\right) u^{m},
\end{gathered}
$$

where $\xi_{k}(t) \equiv \xi_{k}^{e_{k}}(t) \in C^{\infty}\left([0, T], \square^{1}\right)$ arbitrary scalar functions, $k=\overline{1, n}$. Let us now show absolute and uniform convergence of the series (19).

First, we require that there exists a straight line $(\pi)$, passing through zero $\lambda=0$ of the complex plane $\lambda$, such that all $\lambda_{j}(t)$ (for all $t \in[0, T]$ ) lies in the open half-plane bounded by this straight line. It follows that

$$
\inf _{|m| \geq 2, t \in[0, T]} \mid\left(m, \lambda(t)-\lambda_{j}(t) \mid \equiv p_{j}>0, \quad j=\overline{1, n} .\right.
$$

And then, denoting by $p=\min \left\{p_{1}, p_{2}, \ldots, p_{n}\right\}>0$, we have

$$
\begin{aligned}
& \left|y_{s}^{(m)}(t)\right|=\left|\sum_{j=1}^{n} \frac{\left(h^{(m)}(t), d_{j}(t)\right)}{(m, \lambda(t))-\lambda_{j}(t)} c_{s j}(t)\right| \leq \sum_{j=1}^{n} \frac{\left|\left(h^{(m)}(t), d_{j}(t)\right)\right|}{\left|(m, \lambda(t))-\lambda_{j}(t)\right|}\left|c_{s j}(t)\right| \leq \\
& \leq \frac{1}{p} \sum_{j=1}^{n} \sum_{k=1}^{n}\left|h_{k}^{(m)}(t)\right|\left|d_{k j}(t)\right|\left|c_{s j}(t)\right| \leq \sum_{j=1}^{n}\left|h_{k}^{(m)}(t)\right|,
\end{aligned}
$$

(here $c_{s j} s$ - th component of the vector $c_{j}, d_{k j}, h_{k}^{(m)} k$-th components of the vectors $d_{j}$ and $h^{(m)}$ respectively, $c=\sum_{j=1}^{n} \max _{t \in[0, T]}\left|d_{k j}(t)\right| \cdot\left|c_{s j}(t)\right| . \quad$ Since the series $\sum_{|m| \geq 2}\left|h_{k}^{(m)}(t)\right||u|^{m} \quad$ converge uniformly with respect to $t \in[0, T](h(t, u) \in U)$, then the series $\sum_{|m| \geq 2}\left|y_{s}^{(m)}(t)\right||u|^{m}$ also has the same property (due to the inequalities (21)). Therefore, the function (19) belongs to the space $U$. Theorem 1 is proved.

Thus, in the conditions (12) the system (11) has countless solutions (19), where $\xi_{k}(t)$ - arbitrary scalar functions, $k=\overline{1, n}$. To calculate them, it is necessary to impose additional restrictions on the solution $y(t, u)$ of system (11).

Theorem 2. Let the conditions 1), 2a) - 2b) hold, and the right hand side $h(t, u) \in U$ satisfies conditions (12). Then the problem (11) with additional restrictions:

$$
<-\sqrt{t} \frac{\partial y^{(1)}(t, u)}{\partial t}+B(t) y^{(1)}(t, u)+v^{(1)}(t, u), v_{j}(t, u)>\equiv 0 \forall t \in[0, T, j=\overline{1, n},
$$

where $B(t) \in C^{\infty}\left([0, T], \square^{n}\right)$ known $n \times n$ matrix, $v^{(1)}(t, u) \in U^{(1)}$ known vector function, uniquely solvable in the space $U$. 
Proof. Subjecting the solution (19) to the initial condition $y(0, \overline{1})$; we will have:

$$
\sum_{k=1}^{n} \xi_{k}(0) c_{k}(0)=y^{*}-\sum_{k=1}^{n} \sum_{j \neq k, j=1}^{n} \frac{\left(h^{e_{k}}(0), d_{j}()\right)}{\lambda_{k}(0)-\lambda_{j}(0)} c_{j}(0)+\sum_{|m| \geq 0,|m| \neq 1}\left(\sum_{j=1}^{n} \frac{\left(h^{(m)}(0), d_{j}(0)\right)}{(m, \lambda(0))-\lambda_{j}(0)} c_{j}(0)\right) \equiv y_{*},
$$

(here we denote the well-known right-hand side by $y_{*}$ ). Multiplying this equality by $d_{j}(0)$ and taking into account biortormality of the systems $\left\{c_{j}(t)\right\}$ and $\left\{d_{k}(t)\right\}$, we find $\xi_{j}(0)=\left(y_{*}, d_{j}(0)\right), j=\overline{1, n}$. Nowsubject the solution (19) to the condition (22). Calculatefirst

$$
-\sqrt{t} \frac{\partial y^{(1)}(t, u)}{\partial t}+B(t) y^{(1)}(t, u)+v^{(1)}(t, u)=\sum_{k=1}^{n}\left[-\sqrt{t} \dot{y}^{e_{k}}(t)+B(t) y^{e_{k}}(t)\right] u_{k}+\sum_{k=1}^{n} v^{e_{k}}(t) u_{k} .
$$

Multiplying this function by $\left.d_{s}(t)\right) u_{s}$, we write the condition $(22)$ in the form:

$$
-\dot{\xi}_{s}(t)-\left[\left(\dot{c}_{s}(t), d_{s}(t)\right)-\left(B c_{s}(t), d_{s}(t)\right)\right] \xi_{s}(t)=l_{s}(t), \quad s=\overline{1, n},
$$

where $l_{s}(t)$ known function in the class $C^{\infty}\left([0, T], \square^{1}\right)$. Joining to these equations the initial conditions $\xi_{s}(0)=\left(y_{*}, d_{s}(0)\right), s=\overline{1, n}$, found earlier, we calculate uniquely the functions $\xi_{s}(t)$ :

$$
\xi_{s}(t)=\left(y_{*}, d_{s}(0)\right) e^{\int^{t} \frac{\left[B(s) c_{s}(s)-\dot{c}_{s}(s)\right]}{\sqrt{s}} d s}-\int_{0}^{t} e^{\int^{t} \frac{\left[B(s) c_{s}(s)-\dot{c}_{s}(s)\right]}{\sqrt{s}} d s} l_{s}(x) d x, \quad s=\overline{1, n}
$$

and thus, uniquely we find the solution (19) of the problem (11). Theorem 2 is proved.

Example 1. Let in the system

$$
\begin{array}{ll}
\varepsilon y^{(1 / 2)}=\lambda_{1} y-\varepsilon \varphi^{\prime}(t), & y(0, \varepsilon)=y^{0}, \\
\varepsilon z^{(1 / 2)}=\lambda_{2} z+\varepsilon y^{2}-\varepsilon g^{\prime}(t), & z(0, \varepsilon)=z^{0} .
\end{array}
$$

eigennumbers $\lambda_{1}$ and $\lambda_{2}$ are constant and related by $\lambda_{2}=2 \lambda_{1}, \quad \lambda_{1} \neq \lambda_{2}, \quad \operatorname{Re} \lambda_{i} \leq 0, \quad \lambda_{i} \neq 0, \quad i=1,2$. Equivalent problem has the following form:

$$
\begin{array}{ll}
\varepsilon \sqrt{t} y^{\prime}=\lambda_{1} y-\varepsilon \varphi^{\prime}(t), & y(0, \varepsilon)=y^{0}, \\
\varepsilon \sqrt{t} z^{\prime}=\lambda_{2} z+\varepsilon y^{2}-\varepsilon g^{\prime}(t), & z(0, \varepsilon)=z^{0} .
\end{array}
$$

Introduce regularizing variables:

$$
\tau_{1}=-\frac{2 \lambda_{1}}{\varepsilon} \sqrt{t} \equiv \varphi_{1}(t, \varepsilon), \quad \tau_{2}=\frac{2 \lambda_{2}}{\varepsilon} \sqrt{t} \equiv \varphi_{1}(t, \varepsilon) .
$$

For extended functions $\tilde{w} \equiv\{\tilde{y}(t, \tau, \varepsilon), \tilde{z}(t, \tau, \varepsilon)\}$ we obtain the following problem:

$$
\begin{array}{ll}
\varepsilon \sqrt{t} \frac{\partial \tilde{y}}{\partial t}+\lambda_{1} \frac{\partial \tilde{y}}{\partial \tau_{1}}+\lambda_{2} \frac{\partial \tilde{y}}{\partial \tau_{2}}-\lambda_{1} \tilde{y}=-\varepsilon \varphi^{\prime}(t), & \tilde{y}(0,0,0, \varepsilon)=y^{0}, \\
\varepsilon \sqrt{t} \frac{\partial \tilde{z}}{\partial t}+\lambda_{1} \frac{\partial \tilde{z}}{\partial \tau_{1}}+\lambda_{2} \frac{\partial \tilde{z}}{\partial \tau_{2}}-\lambda_{2} \tilde{z}=\varepsilon \tilde{y}^{2}-\varepsilon g^{\prime}(t), & z(0,0,0, \varepsilon)=z^{0} .
\end{array}
$$

Defining a solution of this problem in the form of series

$$
\tilde{y}(t, \tau, \varepsilon)=\sum_{k=0}^{\infty} \varepsilon^{k} y_{k}(t, \tau), \quad \tilde{z}(t, \tau, \varepsilon)=\sum_{k=0}^{\infty} \varepsilon^{k} z_{k}(t, \tau) .
$$

we get the following iteration systems:

$$
\begin{aligned}
& L_{0} y_{0}(t, \tau) \equiv \lambda_{1} \frac{\partial y_{0}}{\partial \tau_{1}}+\lambda_{2} \frac{\partial y_{0}}{\partial \tau_{2}}-\lambda_{1} y_{0}=0, \quad y_{0}(0,0,0)=y^{0}, \\
& L_{0} z_{0}(t, \tau) \equiv \lambda_{1} \frac{\partial z_{0}}{\partial \tau_{1}}+\lambda_{2} \frac{\partial z_{0}}{\partial \tau_{2}}-\lambda_{2} z_{0}=0, \quad z_{0}(0,0,0)=y^{0}, \\
& L_{0} y_{1}(t, \tau)=-\sqrt{t} \frac{\partial y_{0}}{\partial t}-\varphi^{\prime}(t), \quad y_{1}(0,0,0)=0, \\
& L_{0} z_{1}(t, \tau)=-\sqrt{t} \frac{\partial z_{0}}{\partial t}+y_{0}^{2}-g^{\prime}(t), z_{1}(0,0,0)=0,
\end{aligned}
$$


The problem (24) in the space $U$ has a solution

$$
y_{0}(t, \tau)=\alpha_{1}(t) e^{\tau_{1}}, \quad z_{0}(t, \tau)=\alpha_{2}(t) e^{\tau_{2}},
$$

where $\alpha_{k}(t)$ arbitrary functions, $k=1,2$. We find initial data for the functions $\alpha_{i}(t): \alpha_{1}(0)=y^{0}, \alpha_{2}(0)=z^{0}$.

Now we construct the problem (25). It will have the form:

$$
\begin{aligned}
& L_{0} y_{1}(t, \tau)=-\sqrt{t} \alpha_{1}^{\prime}(t) e^{t_{1}}-\varphi^{\prime}(t), \\
& L_{0} z_{1}(t, \tau)=-\sqrt{t} \alpha_{2}^{\prime}(t) e^{t_{2}}+\alpha_{1}^{2}(t) e^{2 t_{1}}-g^{\prime}(t), z_{1}(0,0,0)=0 .
\end{aligned}
$$

For solvability of (27) in the space $U$ we need the following system of equations: $-\sqrt{t} \alpha_{1}^{\prime}=0, \alpha_{1}(0)=y^{0},-\sqrt{t} \alpha_{2}^{\prime}=-\alpha_{1}^{2}, \alpha_{2}(0)=z^{0}$, where we find $\alpha_{1}(t)=y^{0}, \alpha_{2}(t)=z^{0}+2 y_{0}^{2} \sqrt{t}$, and thus, the functions $y_{0}(t, \tau)$ and $z_{0}(t, \tau)$ will be:

$$
y_{0}(t, \tau)=y^{0} e^{\tau_{1}}, z_{0}(t, \tau)=z^{0} e^{\tau_{2}}+2 y_{0}^{2} \sqrt{t} e^{\tau_{2}} .
$$

Example 2. Find approximate solutions and build graphs of the system solution.

$$
\varepsilon\left(\begin{array}{l}
y^{(1 / 2)}(t, \varepsilon) \\
z^{(1 / 2)}(t, \varepsilon)
\end{array}\right)=\left(\begin{array}{ll}
0,2 & 0 \\
0,1 & 1
\end{array}\right)\left(\begin{array}{l}
y(t, \varepsilon) \\
z(t, \varepsilon)
\end{array}\right)+\left(\begin{array}{cc}
0,1 \cdot y \cdot z \\
0,1 \cdot z^{2}
\end{array}\right), \begin{aligned}
& y(0,1)=1, \\
& z(0,1)=1,
\end{aligned}
$$

for different values $\varepsilon$ in the environment of the computer mathematical system Maple [7-8]. $>$ restart; cond: $=\mathrm{y}(0.1)=1, \mathrm{z}(0.1)=1$;

$$
\text { cond }:=\mathrm{y}(0.1)=1, \mathrm{z}(0.1)=1
$$

> sys:=diff( $(0.1 * \mathrm{y}(\mathrm{t}), \mathrm{t})=(\operatorname{sqrt}(2) * \mathrm{y}(\mathrm{t})+0.1 * \mathrm{y}(\mathrm{t}) * \mathrm{z}(\mathrm{t})) / \operatorname{sqrt}(\mathrm{t}),(\operatorname{diff}(0.1 * \mathrm{z}(\mathrm{t}), \mathrm{t})=(\mathrm{z}(\mathrm{t})+0.1 *$ $(\mathrm{y}(\mathrm{t})+\mathrm{z}(\mathrm{t}) * \mathrm{z}(\mathrm{t}))) / \operatorname{sqrt}(\mathrm{t}))$

$$
\begin{aligned}
& \text { sys }:=0.1\left(\frac{d}{d t} \mathrm{y}(t)\right)=\frac{\sqrt{2} \mathrm{y}(t)+0.1 \mathrm{y}(t) \mathrm{z}(t)}{\sqrt{t}}, 0.1\left(\frac{d}{d t} \mathrm{z}(t)\right)=\frac{\mathrm{z}(t)+0.1 \mathrm{y}(t)+0.1 \mathrm{z}(t)^{2}}{\sqrt{t}} \\
& \text { sys }:=0.2\left(\frac{d}{d t} \mathrm{y}(t)\right)=\frac{\sqrt{2} \mathrm{y}(t)+0.2 \mathrm{y}(t) \mathrm{z}(t)}{\sqrt{t}}, 0.2\left(\frac{d}{d t} \mathrm{z}(t)\right)=\frac{\mathrm{z}(t)+0.2 \mathrm{y}(t)+0.2 \mathrm{z}(t)^{2}}{\sqrt{t}}
\end{aligned}
$$

$>\mathrm{F}:=$ dsolve $(\{$ sys, cond $\},[\mathrm{y}(\mathrm{t}), \mathrm{z}(\mathrm{t})]$, numeric $)$ :

$>$ with(plots):

$>\mathrm{p} 1:=\operatorname{odeplot}(\mathrm{F},[\mathrm{t}, \mathrm{y}(\mathrm{t})], 0 . .0 .1$, color=red,thickness=2, linestyle $=3)$ :

$>\mathrm{p} 2:=\operatorname{odeplot}(\mathrm{F},[\mathrm{t}, \mathrm{z}(\mathrm{t})], 0 . .0 .1$, color $=$ green,thickness $=2)$ :

$>$ p3:=textplot $([0.1,0.6, " y(t) "]$, font=[TIMES,ITALIC, 12]):

$>$ p4:=textplot([0.08,0.6,"z(t)"], font=[TIMES,ITALIC, 12]):

$>\operatorname{display}(\mathrm{p} 1, \mathrm{p} 2, \mathrm{p} 3, \mathrm{p} 4)$
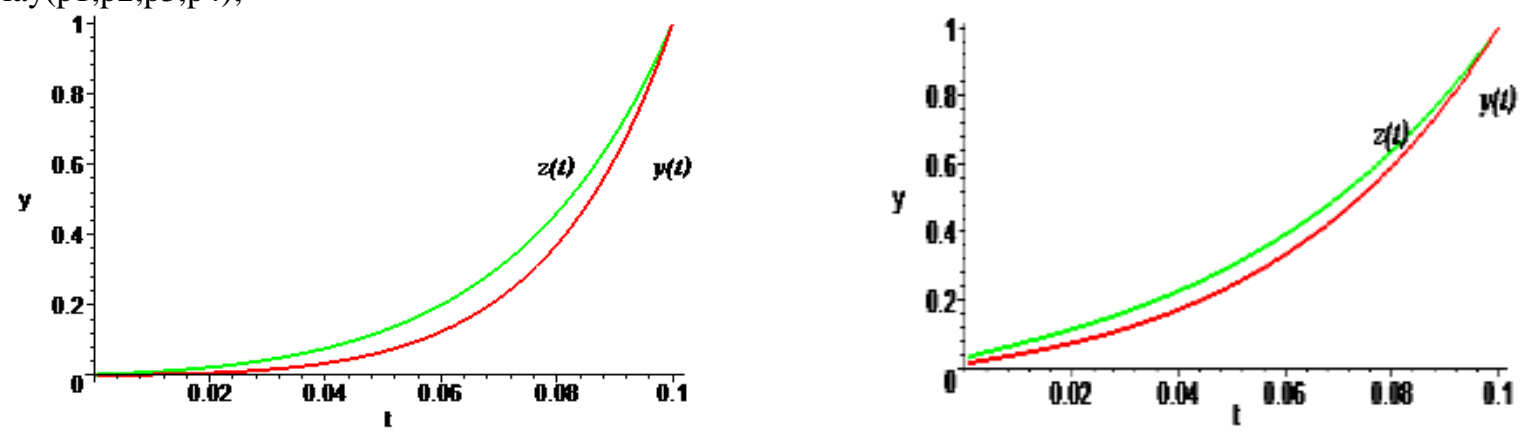

Acknowledgments. This work is supported by the grant AP05133858 "Contrast structures in singularly perturbed equations and their application in the theory of phase transitions" by the Scientific Committee of the Ministry of Education and Science of the Republic of Kazakhstan.

\section{REFERENCES}

[1] Safonov, V.F. (1987) Multipoint resonance in a strongly nonlinear singularly perturbed system of differential equations. J. Differential Equations, 3, 529-530.

[2] Safonov, V.F. (1989) Normal forms and regularization of nonlinear singularly perturbed evolution equations. J. Differential Equations, 25, 627-635.

[3] Safonov, V.F. (1979) Regularization method for singularly perturbed systems of nonlinear differential equations. Izv. AS of USSR. Ser. mathem., 43, (3), 628-653. 
[4] Kalimbetov B.T. (2007) Algorithm of method of normal forms for a weakly nonlinear equation with an unstable spectrum. Uzbek. Mathem. Journal, 2, 56-69.

[5] Kalimbetov, B.T. and Safonov, V.F. (1989) Normalized asymptotic solutions of weakly nonlinear singularly perturbed Cauchy problem with an unstable spectrum. Research on Integro-Differential. Equation, Frunze: "Ilim", 22 , 37-42.

[6] Dyakonov, V.P. Maple 7. Training Course. St. Petersburg, "Peter", 2003.

[7] Kirsanov, M.N. Graphs in Maple. Moscow, Fizmathlit, 2007. 